\title{
Optical Filter Design for the Fluorescence Detector of the AUGER Project
}

\author{
E. Fokitis, S. Maltezos, and E. Papantonopoulos \\ Physics Department, \\ National Technical University of Athens, \\ Zografou Campus, 15780 Athens, Greece
}

Abstract: The design concept of the required optical filters for the Fluorescence Detector of the AUGER Project, is presented in this work. Due to the needed high transmittance values in the UV region, the dielectric thin film multilayer technique, using UV-transparent materials, has been selected. The design method applied, is the "Monte Carlo Simulated Annealing" (MOCASA). Two alternative target spectral transmittance curves have been considered, as candidate for the optical filters. These are, the single-band pass and the multiband pass type, both covering the atmospheric nitrogen fluorescence spectral range. The design performance calculation, is based on the expected signal and the moonless night-sky background photon rates, selected by the optical filters. Characterizations of simple and double layres, using various candidate materials, are in progress, planning to produce a full prototype in near future.

\section{Introduction}

The design and construction of optical filters for the photomultipliers (PMT's) of the Fluorescence Detector, is of primary importance for the Auger Project [i.1.1. The optical filter, placed in front of each light collector and PMT, is indispensable in order to reduce the night sky background light, maximizing the ratio of signal photon to background photon rate. The Extensive Air Shower (EAS), which is developed by the interaction of very energetic cosmic ray with the atmospheric air, is a swarm of particles moving at the speed of light through the atmosphere. They ionize and excite nitrogen atoms, which radiate nearUV photons. The light spectrum of the nitrogen fluorescence covers mainly the range $300-400 \mathrm{~nm}$. The Fluorescence Detector sees the shower as a spot of light, whose motion is along the shower axis. The light is collected and detected by a Collector/PMT/Optical Filter array. The principle of operation of the Fluorescence Detector is shown in the Fig. $\underline{1}_{r}^{1}$

The direction of the shower axis is the cosmic ray's arrival direction. A geometrical reconstruc- tion is required to determine the shower axis. The three Fluorescence Detectors in each hemisphere will cover 360 in azimuth and 30 in elevation angle. Each Detector will include 12 spherical mirrors for imaging the fluorescence light.

The required high transmittance of the optical filters in UV region, leads to specific techniques, as the dielectric multilayer thin film deposition on a substrate by using high/low index UV-transparent materials. The optimal solution, is defined as the configuration of the minimum number of layers which build an optical filter and have a spectral transmittance converging to the target one. The calculated overall error in each case, has to be minimized and to be between the allowed predefined constraints. The method to calculate the thickness of each layer in order to obtain the optimal solution, is presented in this work.

\section{The need of optical filters and their specifications}

We need the optical filters to achieve a sufficiently high signal to noise ratio ( $\mathrm{S} / \mathrm{N}$ ratio) of 


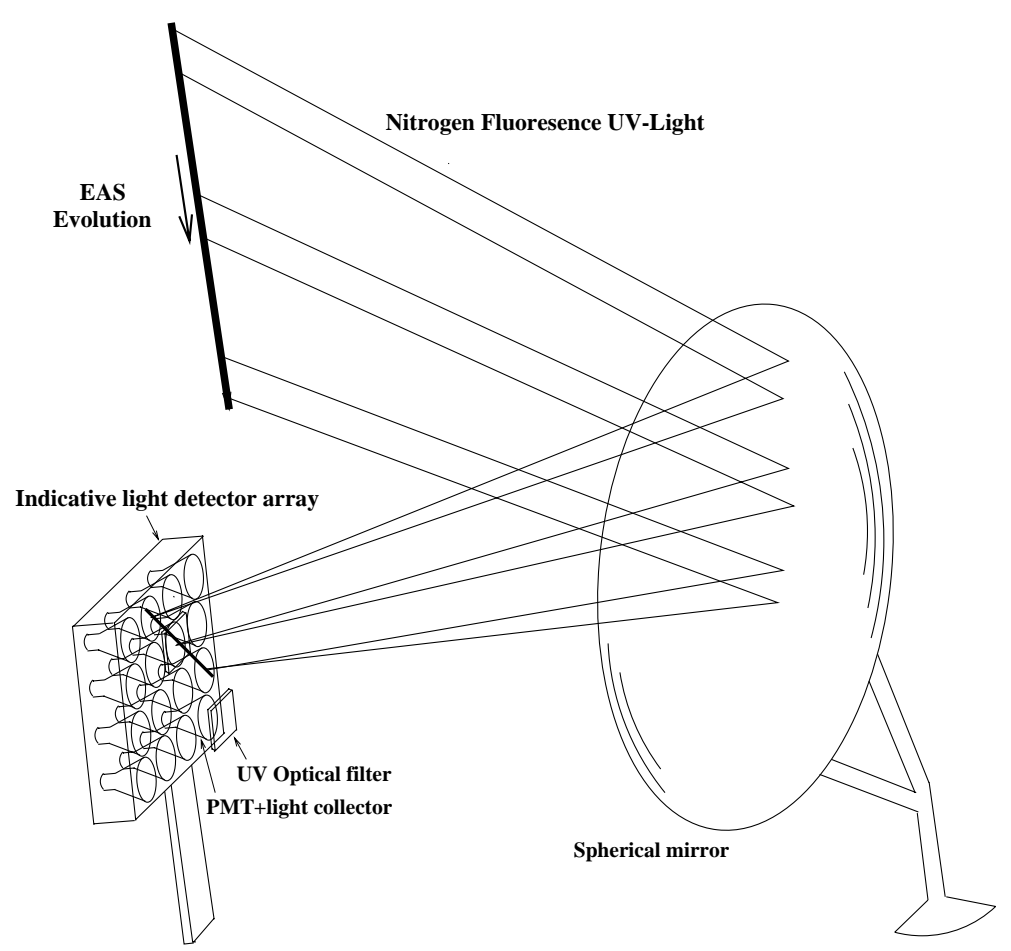

Figure 1: The principle of the Fluorescence Detector. The linear evolution of the EAS is imaging in the focal plane of the mirror.

PMT photoelectron signal, in order to detect air showers even from long distances. Optical filters also could extent the operation cycle of the fluorescence detector during moon presence, where its direct or diffused light produces high levels of background noise. High $\mathrm{S} / \mathrm{N}$ ratio values could be achieved if the transmittance in the stop-band tends to zero.

An extra reduction of the background is based on the idea to have a multipeak pass-band, matching with the nitrogen spectrum. In this case, we have to design optical filters of the multiband pass type. Designing optical filters for the fluorescence detector, we must take into account the following main functional and technical constraints:

1. High Transmittance in the region $300-420$ $\mathrm{nm}$ in order to improve in combarison with the performance of absorption type filters used up to now in similar experiments : > $85 \%$ in average.

2. Very Low Transmittance in the region 420$600 \mathrm{~nm}: \simeq 2 \%$ or less in average.
3. To maintain the selection of the fluorescence signal within a RMS variation of incidence angle up to $15^{\circ}$.

4. Uniform spectral characteristics achievement and mechanical stability in the mass production.

\section{The method : Simulated Anneal- ing}

In trying to find an optimum multilayer filter which satisfies a pre-defined spectral transmittance, the main problem is the appearance of many local minima in the so-called Merit which is the function to be minimized. This function has as a variable the wavelength $\lambda$ and as parameters the optical thicknesses of the layers $\left(d_{1}, d_{2}, \ldots, d_{N}\right)$, where $\mathrm{N}$ is the total number of the layers. Its mathematical expression in discrete form is :

$$
\Delta M=\sqrt{\frac{1}{m} \sum_{i=1}^{m}\left[\frac{T_{c}\left(\lambda_{i}\right)-T_{t}\left(\lambda_{i}\right)}{\delta T_{t}\left(\lambda_{i}\right)}\right]^{2}}
$$


where $T_{c}$ is the calculated and $T_{t}$ is the target spectral transmittance, $\delta T_{i}$ is an assumed uncertainty of $T_{t}$ and $\mathrm{m}$ is the total number of computation steps. The $T(\lambda)$ extraction is based on the numerical computation of the elements of the resulting characteristic matrix of the multilayer, according to the thin film theory. The use of local minimization algorithms is efficient only if we have a good starting solution. For this reason we have further developed the simulatedannealing method $[\overline{2}, 1, \overline{6}$. This method uses the "Metropolis" algorithm [ịi algorithm that generates a sequence of states converging asymptotically towards the Boltzman distribution. Hence the system reaches at the limit a thermodynamic equilibrium at temperature $\mathrm{T}$.

It begins in a arbitrary state and successively generates candidate state transitions at random for the temperature T. Each such elementary transition corresponds to a small change in the global energy E of the system (correspoding to the Merit Function in our case). More specifically the method of simulated annealing can be expressed in a numerical algorithm which can be described by the following steps:

1. Start with initial temperature $T$ such that the system (which is our filter) is completely disorganized.

2. Make a small change in our system by a random variation of the filter parameters.

3. Evaluate the resulting change in energy (Merit Function).

4. Accept the new filter with the propability given by the Metropolis criterion, which is described as :

If $\Delta E<0$ accept the filter.

If $\Delta E \geq 0$, find a random number $r$ between 0 and 1 .

If $\exp (-\Delta E / T)>r$, accept the filter, otherwise reject it.

5. Repeat the steps (2)-(4) until the system reaches quasi-equilibrium for the given temperature.

6. Perform a zero temperature steepest quench using exponential dependence.

\section{Go to step (2)}

8. Terminate the procedure if the temperature becomes very low or the acceptance rate tends to zero.

In order to reduce the run time, we further improved the method introducing some quantities to control the algorithm during running. In particular, we calculate the "acceptance rate" (AR) of the transitions periodically and we adjust the step of the thickness variation (step range) according to the AR level.

This method has been implemented and further developed using a macro and FORTRAN functions in the PAW [i] $[\overline{1}]$ environment.

\section{The design parameters}

In order to achieve an optimized multilayer filter design for the Fluorescence Detector, we defined two alternative types of the required target spectral transmittance to be satisfied :

a) A Single-Band Pass (SBP), within the nitrogen spectrum range.

b) A Multi-Band Pass (MBP), which matches the main nitrogen emission lines.

The latter is expected to be efficient in the case of Moonlight and artificial light presence or in the case of Cherenkov radiation from EAS. In the so-called non-quarterwave multilayer technique [10 1 , each layer has its independed optical thickness value, and in general, the corresponding optical thickness differs from $\lambda / 4$. The considered multilayer has the structure shown in the Fig. $\overline{2}_{2}^{1}$ Pairs of the candidate UV-transparent dielectric materials, as $\mathrm{HfO}_{2} / 0.098 \mathrm{Y}_{2} \mathrm{O}_{3}, \mathrm{Al}_{2} \mathrm{O}_{3}$, $\mathrm{Sc}_{2} \mathrm{O}_{3}$ and $\mathrm{ZrO}_{2}$ for high refractive index and $\mathrm{MgF}_{2}, \mathrm{SiO}_{2}$ for low refractive index, have been used for the design. Also we included their fitted dispersion curves using 2 to 4 term of Sellmeier

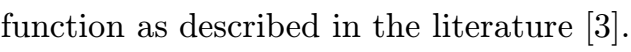

In the design, we considered that each layer is non-absorbing (the extinction coefficient $k$ tends to zero). This assumption is reasonably satisfied in the case of UV-transparent dielectric thin films considered here. 


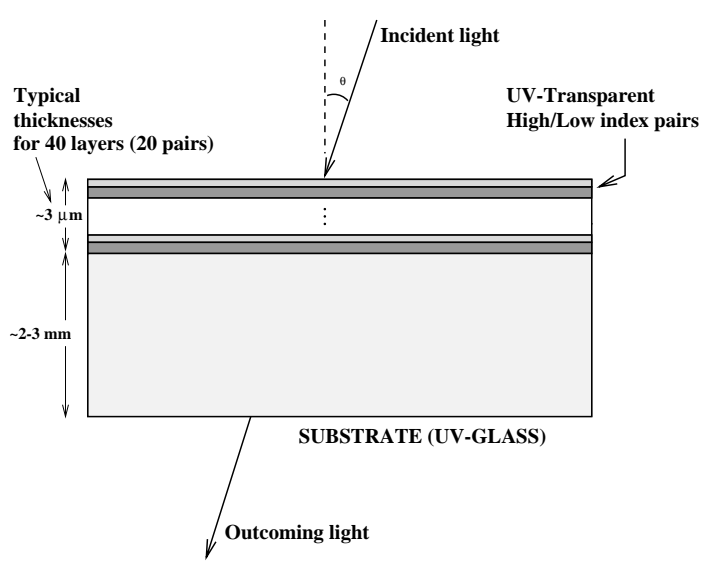

Figure 2: The configuration of a dielectric UVtransparent thin film multilayer filter.

\section{The obtained optical filters and their performances}

In the thin film technique, pairs of materials with high/low refractive index are used, as we mentioned above. We present an application of the design method, using 20 pairs (40 layers) of $\mathrm{HfO}_{2} / 0.098 \mathrm{Y}_{2} \mathrm{O}_{3}$ (for high index) and $\mathrm{MgF}_{2}$ (for low index). on a substrate of fused silica.

As target spectral transmittance, we defined the ideal curves which correspond to the following types of filters :

a) The Single-Band Pass (SBP) filter, where the transmittance is 1 within the region $300-420$ nm and 0 outside.

b) The Multi-Band Pass (MBP) filter, where the transmittance is 1 within the bands which match to the nitrogen spectrum and 0 outside these bands.

The program needs about 8 hours in a Silicon Graphics computer (processor MIPS R4000) to converge to an optimum solution.

The obtained optical filters have been evaluated by calculating the following design performance indicators:

1. Standard deviation, with respect to the target $\sigma=\delta T$.

2. The obtained ratio of signal photon rate to background photon rate : $\frac{N_{s}}{N_{b}}$

The first performance indicator, shows the order of the overall error of the minimization.

The second one, gives us an estimate of the detection efficiency when using the optical filter, in the presence of a specified spectrum of background rate.

The calculation of the above ratio, is based on the following methodology :

Let assume that,

$N_{s}$ and $N_{b}$ are the photon rates selected by the filter for both the signal and the background respectively.

$N_{s i}(\lambda)$ and $N_{b i}(\lambda)$ are the spectra of incident light, within the wavelength range of interest $\Delta \lambda$.

$N_{s t}$ and $N_{b t}$ are the total photon rates incident to the filter.

$N_{s n}(\lambda)$ and $N_{b n}(\lambda)$ are the normalized spectra.

Then, the collected signal spectrum is expressed as :

$$
N_{s c}(\lambda)=N_{s t} \cdot N_{s n}(\lambda)
$$

where

$$
N_{s t}=\int_{\Delta \lambda} N_{s i}(\lambda) d \lambda
$$

And the collected background spectrum is expressed as :

$$
N_{b c}(\lambda)=N_{b t} \cdot N_{b n}(\lambda)
$$

where

$$
N_{b t}=\int_{\Delta \lambda} N_{b i}(\lambda) d \lambda
$$

So, the selected signal rate by the filter of spectral transmittance $T(\lambda)$, can be obtained from :

$$
N_{s s}=N_{s t} \int_{\Delta \lambda} T(\lambda) N_{s n}(\lambda) d \lambda
$$

where

$$
\int_{\Delta \lambda} N_{s n}(\lambda) d \lambda=1
$$

And the selected background rate can be obtained from :

$$
N_{b s}=N_{b t} \int_{\Delta \lambda} T(\lambda) N_{b n}(\lambda) d \lambda
$$

where

$$
\int_{\Delta \lambda} N_{b n}(\lambda) d \lambda=1
$$



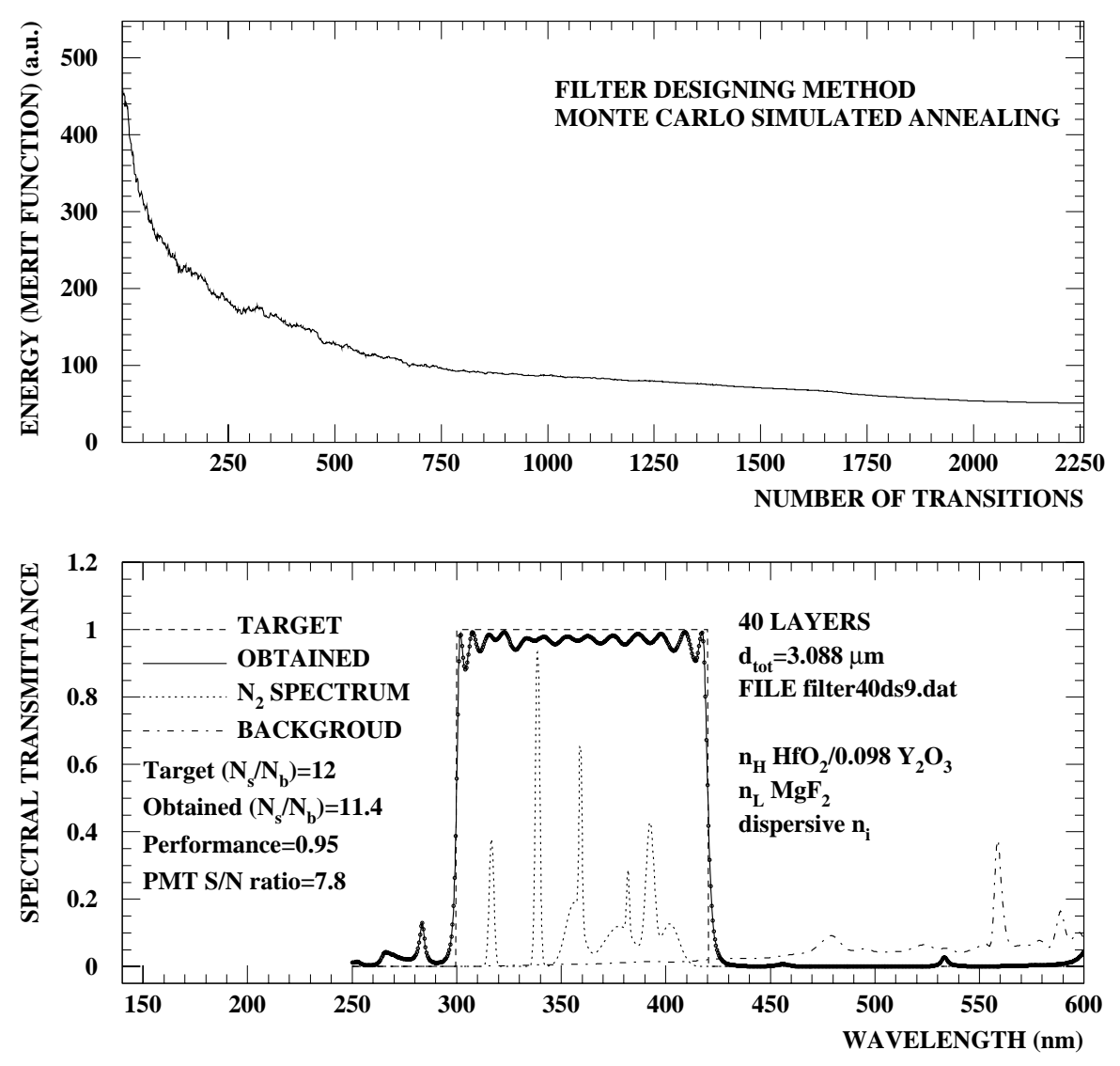

Figure 3: The obtained Single-Band Pass (SBP) filter. In the upper diagram, the evolution of the merit function is plotted. In the lower diagram the obtained curves and characteristics are presenred.

The ratio of the selected signal and Background is written :

$$
\frac{N_{s s}}{N_{b s}}=\frac{N_{s t}}{N_{b t}} \frac{\int_{\Delta \lambda} T(\lambda) N_{s n}(\lambda) d \lambda}{\int_{\Delta \lambda} T(\lambda) N_{b n}(\lambda) d \lambda}=\frac{N_{s t}}{N_{b t}} \frac{N_{s}}{N_{b}}
$$

The fraction $N_{s} / N_{b}$, is the selection performance of the optical filter.

The fraction $N_{s t} / N_{b t}$, is the total signal to total Background rate. This fraction is expected to vary due to change either the signal or to the background.

A typical value concerning the collected photons within $0.5 \mu \mathrm{s}$, taken from the [1]1]

$$
\frac{N_{s s}}{N_{b s}}=\frac{353 p h / \mu s}{150 p h / \mu s}=2.35
$$

The obtained transmittances and the corresponding performances according to the above analysis, are shown in Fig. $\overline{3}$, and $\overline{4}$. The signal (nitrogen fluorescence spectrum) curve has been plotted using the interpolated samples from an experimental measurement of the spectrum of a nitrogen descharged lamp in NTUA. The background curve has been plotted using the interpolated samples from the experimental spectrum of the night-sky background radiation at La Palma [is], combined and normalized with data from Galaxy spectrum, taken by the SAO FAST Spectograph. In order to be visible in the figures, the background total energy was multiplied by 2 . The signal to noise ratio of the PMT (included the filter) has been calculated using the formulas 

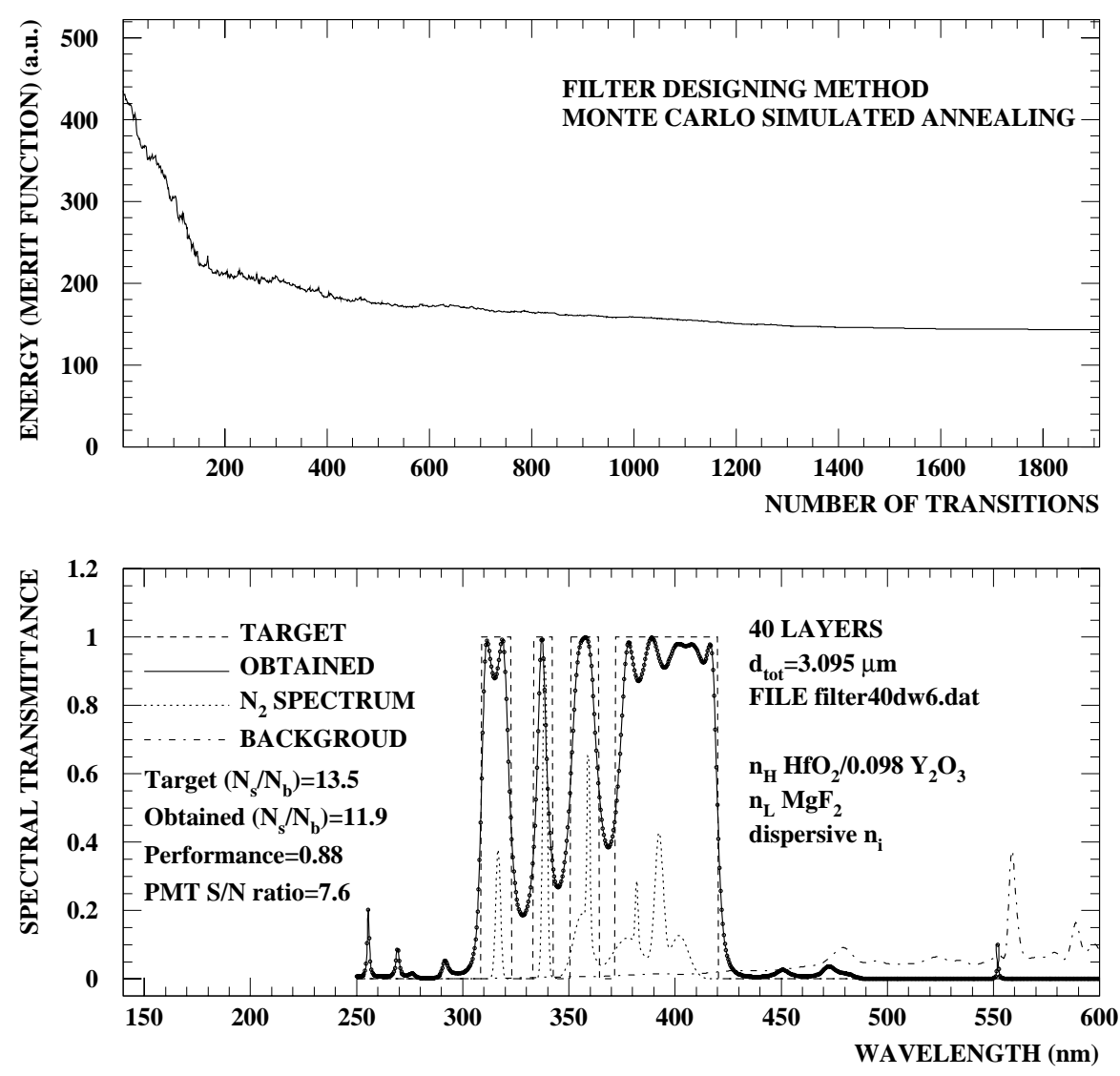

Figure 4: The the obtained Multi-Band Pass (MBP) filter. In the upper diagram, the evolution of the merit function is plotted. In the lower diagram the obtained curves and characteristics are presented.

described in the ref. $[\overline{1} 1]$.

Below we summarize the performances of the above filters assuming for simplicity zero losses at the substrate surfaces.

- Standard deviation : $T=0.005$ in the region $420-600 \mathrm{~nm}$ and $T=0.08$, in the region $<300 \mathrm{~nm}$.

- Signal/Background rate : 11.4/11.9 (With target or ideal filter : 12.0/13.5)

- Performance (in respect to the target one) : $0.95 / 0.88$

- Spectrum shift, towards to the lower wavelength, due to incidence angle variation : $\simeq 8-10 \mathrm{~nm}$.
It is interesting to compare with the corresponding performances of other type of filters, such as absorption filters. However, this will be the topic of further work.

\subsection{The effect of incidence angle variation}

In the design algorithm we have assumed a vertical incidence as a reference case. When the incidence angle differs from zero, the p-polarization and s-polarization must be taken into account when calculating the spectral transmittance. to study its effect The incident light to the photomultiplier collector comes from directions which could vary within $15^{\circ}$ in RMS, from its geometrical axis. So, this variation causes an important effect which we studied. 


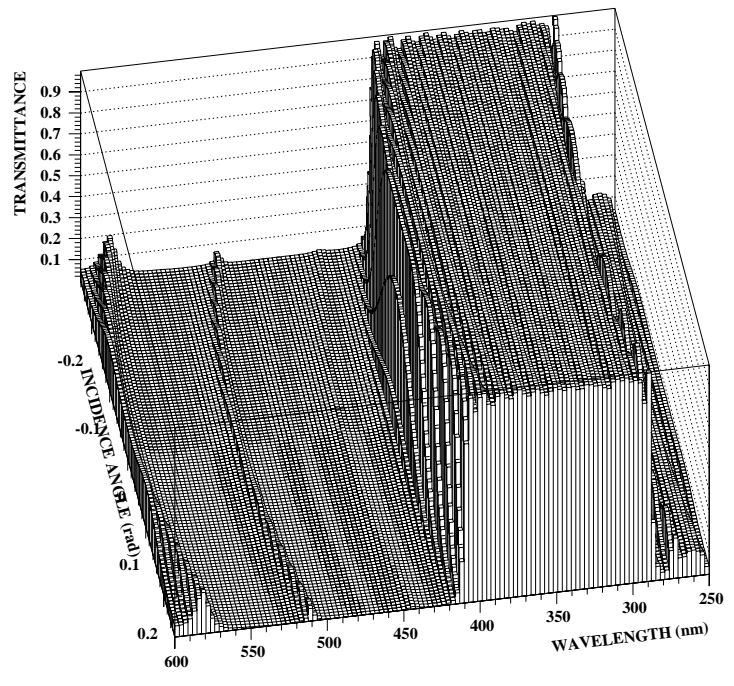

Figure 5: A 3-D representation of the spectral transmittance versus wavelength and incidence angle, where the stability of the whole curve is obvious.

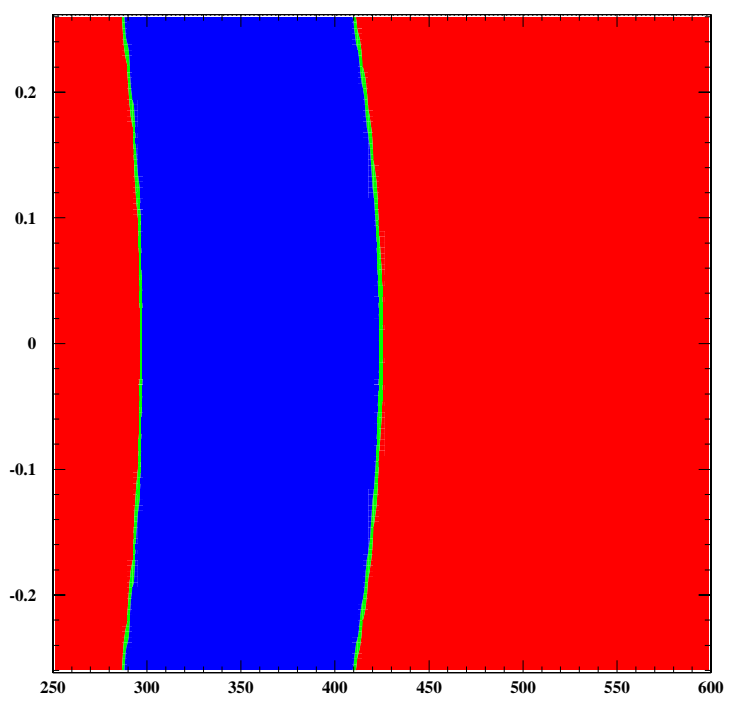

Figure 6: A horizontal contour of the spectral transmittance from Fig.7, showing the effect of shifting, due to the variation of the incidence angle. The dark region corresponds to high transmittance values. The $\mathrm{x}$-axis represents the wavelength in $\mathrm{nm}$ and the $\mathrm{y}$-axis the incidence angle in radians.

In Fig. $\underline{\underline{p}}_{1}^{\prime} \mathrm{a} 3-\mathrm{D}$ plot of the spectral transmittance, shows that the effect of the above variation is a shifting to the lower wavelengths. From an horizontal contour of the 3-D curve shown in Fig. ' $\overline{6}$, , the above shift can be measured. We also observe, that it depends on the wavelength range. This conclusion lead us to forsee a wavelength tollerance towards the higher wavelengths, when we define the target transmittance.

\section{Conclusions}

We have presented a resonable method to design optical filters with high performances for the Fluorescence Detector for the AUGER Project. We have discussed the theoretical and practical aspects of the simulated annealing method. This method allows to build a multilayer coating structure which can satisfy the required optical properties of the filter. The methodology to calculate the performance indicators allows the evaluation of each obtained filter. As an application, we presented a design of Single Band-Pass and Multi-Band Pass optical filter in UV region for the Fluorescence Detector.

Experimental deposition tests of 1 to 6 layers of the mentioned materials, are under way using Electron Beam deposition technique, at NCSR "Demokritos" and Flash Evaporation techniques with heating resistance, at NTUA. For characterization of the above coatings we used the ${ }^{12} \mathrm{C}$ ions back-scattering, the Ellipsometry and the $\mathrm{X}$ ray fluorescence. By the aid of the above tests, we plan to produce a 40-layer prototype optical filter in the near future.

\section{References}

[1] AUGER Collaboration, "Design report of the Pierre AUGER Project", March (1997).

[2] T. Boudet et al. "Thin-film designs by simulated annealing" Appl. Opt., vol. 35, No 31 p62196226 (1996).

[3] HANDBOOK OF OPTICS,Vol. II, McGRAWHILL, INC. (1995).

[4] Ke-Ou Peng and Marcel R. de la Fonteijne "Derivatives of transmittance and reflectance for an absorbing multilayer stack" Appl. Opt., vol. 24, No 4 p501-503 (1985).

[5] S. Laux, K. Mann, B. Granitza, U. Kaiser, and W. Richter, "Antireflection coatings for UV radiation obtained by molecular-beam deposition" Appl. Opt., vol. 35, No 31 p6216-6218 (1996).

[6] M. Plischke and B. Bergersen, "Equilibrium Statistical Physics" 2nd Edition, World Scientific. 
[7] R. Brun, O. Couet, C. Vandoni, and P. Zanarini, "Physics Analysis Workstation", CERN Computer Center Program Library.

[8] C. R. Jenkins, and S. W. Unger, "The nightsky spectrum from La Palma", Royal Greenwich Observatory, Technical Note No 82 (1991).

[9] N. Metropolis et al. "Equation of state calculations by fast computing machines", J. Chem. Phys. 21, 1087-1092 (1953).

[10] B. Vidal, A. Fornier, and E. Pelletier, "Optical monitoring of nonquarterwave multilayer filters", Appl. Opt., vol. 17, No. 7 p1038-1047 (1978). 